\title{
Black-blood dynamic contrast-enhanced coronary artery wall MRl: a potential tool for kinetic- modeling-based wall inflammation assessment
}

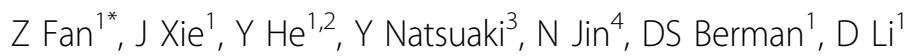 \\ From 16th Annual SCMR Scientific Sessions \\ San Francisco, CA, USA. 31 January - 3 February 2013
}

\section{Background}

Dynamic gadolinium contrast-enhanced (DCE) vessel wall MRI has recently been used to compute a set of model-based contrast kinetic parameters (e.g. $\mathrm{K}^{\text {trans }}$ and $\mathrm{V}_{\mathrm{p}}$ ) that can well characterize the extent of inflammation in carotid plaques $[1,2]$. However, no studies have shown its feasibility in coronary artery wall, presumably due to the technical challenges in imaging such a constantly-moving, ultra-small structure and potential difficulty in visually distinguishing the wall from the hyperintense lumen with conventional DCE techniques. This work aimed to develop a black-blood navigatorgated ECG-triggered T1-weighted sequence for DCE MRI of coronary vessel wall.

\section{Methods}

An SR-DIR (saturation recovery combined with double inversion recovery) preparation is combined with an RF spoiled GRE sequence to achieve: 1) To create T1weighting for vessel wall; 2) To consistently null the blood signal with a fixed inversion time combination (TI1 and TI2) (Fig. 1). Bright-blood acquisition is interleaved with black-blood acquisition to enable arterial blood signal measurement as needed in kinetic modeling [3].

Ten healthy volunteers (1 F, 9 M; age 22-45 years) were scanned at $3 \mathrm{~T}$ using a 6 -channel body matrix coil and spine coil. DCE imaging was performed using the developed technique at one single slice selected from

one of major coronary arteries. Relevant imaging parameters: resolution $=0.8 \times 0.8 \times 4.0 \mathrm{~mm}^{3}$, TI1/TI $2=$ $350 / 40 \mathrm{~ms}$. One-frame pre-contrast scan was followed by repetitive contrast-enhanced scans (1-2 $\mathrm{min} /$ frame, > $15 \mathrm{~min})$, along with intravenous contrast $(0.2 \mathrm{mmol} / \mathrm{kg}$ gadopentetate dimeglumine) injection and saline flush $(30 \mathrm{ml})$ both at $0.2 \mathrm{ml} / \mathrm{s}$. The changes in signal intensity of coronary vessel wall and lumen were obtained from black-blood and bright-blood images, respectively.

\section{Results}

The lumen signal was consistently nulled in the blackblood acquisition (Fig. 2A a, c) and vessel wall was clearly differentiated from the lumen. The sharp wash-in and slow wash-out process of the blood signal was observed in all the volunteers (Fig. 2B). From the 10 subjects, $\mathrm{K}^{\text {trans }}=0.031 \pm 0.020 \mathrm{~min}^{-1}, \mathrm{~K}_{\mathrm{ep}}=0.226 \pm 0.105$ $\min ^{-1}$, and $\mathrm{V}_{\mathrm{p}}=38.37 \pm 23.34 \%$. The kinetic model fitted the acquired data well $(\mathrm{R} 2=0.80 \pm 0.07$, ANOVA analysis).

\section{Conclusions}

With SRDIR preparation, black blood imaging can be fulfilled consistently regardless of blood $\mathrm{T} 1$ value. This could improve the accuracy of vessel wall signal measurement and make this technique feasible for coronary vessel wall that is much thinner than carotid vessel wall. A feasibility study applying this technique to clinical patients with stable angina is underway.

${ }^{1}$ Cedars-Sinai Medical Center, Los Angeles, CA, USA

Full list of author information is available at the end of the article

(C) 2013 Fan et al; licensee BioMed Central Ltd. This is an Open Access article distributed under the terms of the Creative Commons Attribution License (http://creativecommons.org/licenses/by/2.0), which permits unrestricted use, distribution, and reproduction in any medium, provided the original work is properly cited. 


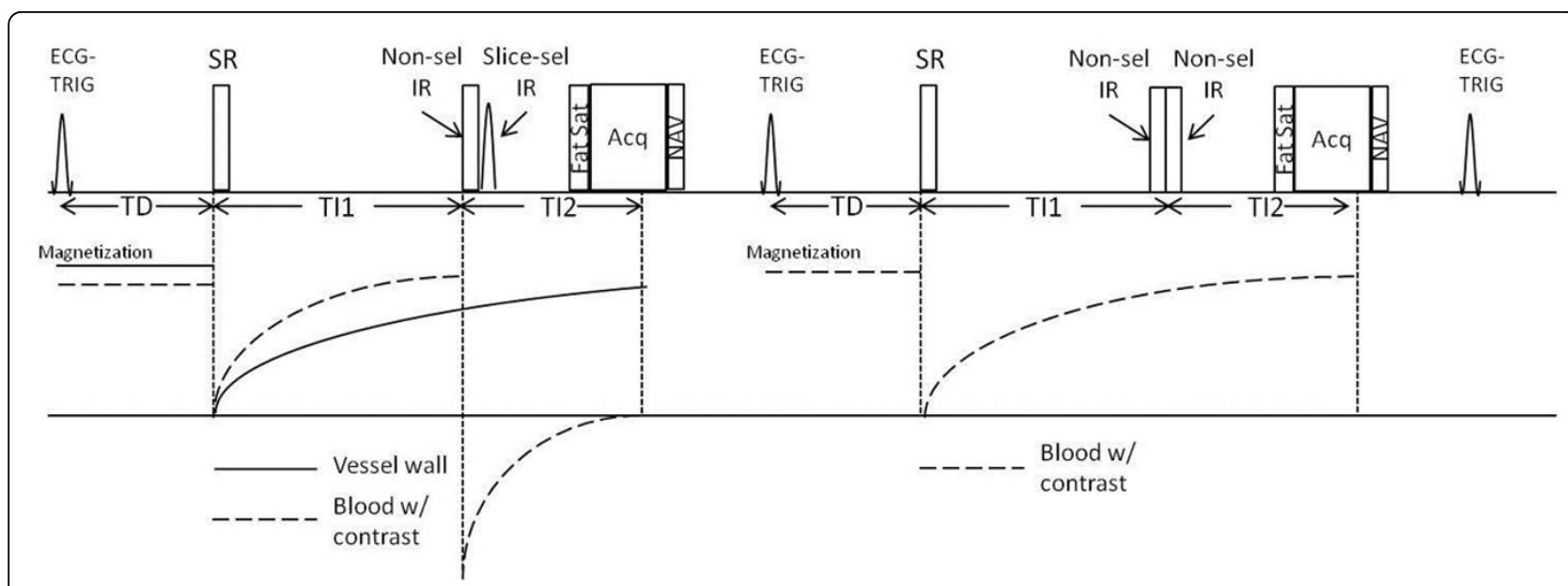

Figure 1 Sequence diagram of the ECG-triggered and navigator-gated SR-DIR prepared RF spoiled GRE sequence. Black-blood and bright-blood images data are collected in an interleaved fashion. Blood signal is suppressed by a combination of SR and dual-IR. SR: saturation recovery; IR: inversion recovery.

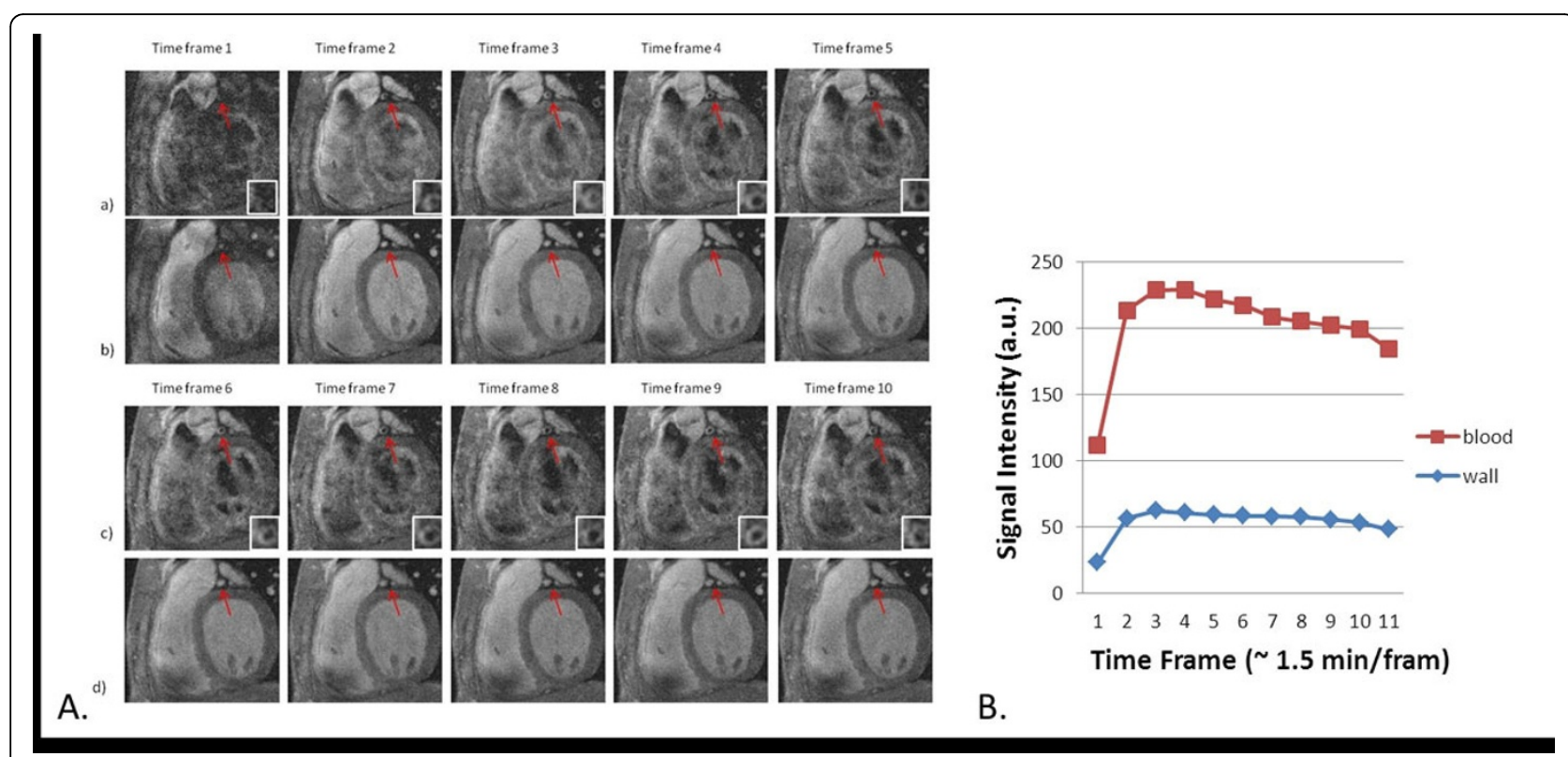

Figure 2 A. Representative dynamic black-blood $(a, c)$ and bright-blood $(b, d)$ images throughout the contrast injection process. B. Typical signal intensity vs. time curves of coronary vessel wall and blood.

\section{Author details}

'Cedars-Sinai Medical Center, Los Angeles, CA, USA. ${ }^{2}$ Radiology, Beijing

Anzhen Hospital, Beijing, China. ${ }^{3}$ Siemens Healthcare, Los Angeles, CA, USA.

${ }^{4}$ Siemens Healthcare, Columbus, OH, USA.

Published: 30 January 2013

doi:10.1186/1532-429X-15-S1-W13

Cite this article as: Fan et al:: Black-blood dynamic contrast-enhanced

coronary artery wall MRI: a potential tool for kinetic-modeling-based

wall inflammation assessment. Journal of Cardiovascular Magnetic

Resonance 2013 15(Suppl 1):W13. 